\title{
A Parallel Robotic System with Force Sensors for Percutaneous Procedures Under CT-Guidance
}

\author{
Benjamin Maurin ${ }^{1}$, Jacques Gangloff ${ }^{1}$, Bernard Bayle ${ }^{1}$, Michel de Mathelin ${ }^{1}$, \\ Olivier Piccin ${ }^{2}$, Philippe Zanne ${ }^{1}$, Christophe Doignon ${ }^{1}$, Luc Soler ${ }^{3}$, and \\ Afshin Gangi ${ }^{4}$ \\ 1 LSIIT ${ }^{\ddagger}$ (UMR CNRS-ULP 7005), Strasbourg I University \\ Bd. S. Brant, BP 10413, 67412 Illkirch cedex, FRANCE \\ maurin@eavr.u-strasbg.fr \\ 2 LICIA(EA3434), INSA-Strasbourg \\ 24, Bd de la Victoire, 67084 Strasbourg, FRANCE \\ 3 IRCAD, Hôpital Civil, \\ 67000 Strasbourg, FRANCE \\ 4 Department of Radiology B., \\ University Hospital of Strasbourg, \\ 67091 Strasbourg, FRANCE
}

\begin{abstract}
This paper presents a new robotic framework for assisted CT-guided percutaneous procedures with force feedback and automatic patient-to-image registration of needle. The purpose is to help practitioners in performing accurate needle insertion while preserving them from harmful intra-operative X-rays imaging devices. Starting from medical requirements for needle insertions in the liver under CT-scan, a description of a dedicated parallel robot is made. Its geometrical and physical properties are explained. The design is mainly based on the accuracy and safety constraints. A real prototype is presented that is currently tested.
\end{abstract}

\section{Introduction}

Percutaneous medical procedures are among the new minimally invasive techniques that are emerging thanks to the progress of medical imaging and medical devices. Their field of application ranges from the diagnosis of pains to the treatments of tumors inside internal organs. This paper mainly focuses on the biopsies of abdominal organs and the radiofrequency ablations of tumors. These interventions are known to be less painful for the patient than a classical surgical act and thus allows faster recovery.

Given the small size of detectable tumors with current imaging devices, these procedures require high precision targeting during insertion. The success rate of such interventions is highly correlated with the accuracy of the needle positioning. In current manual procedures, the needle is hold by the radiologist and

\footnotetext{
$\ddagger$ The authors wish to thank the Alsace Region Council for the financial support of this research project.
} 
a visual guidance is needed, since freehand guidance with direct tactile feedback is not sufficient. For highly precise interventions (about $1 \mathrm{~mm}$ of accuracy) computed tomography has proved to be an excellent imaging modality given its resolution and good tissue differentiation. Furthermore, CT guidance is very useful when critical organ areas have to be bypassed, like the portal vein in the liver. While recent CT-scans allow the detection of tumors of $1 \mathrm{~cm}$ and below, current manual interventions are only made on tumors of 3 to $6 \mathrm{~cm}$. The main reason is that destruction of these tumors with freehand insertion is not possible due to accuracy problems. It should be noted also that, during a CT-guided needle insertion, the interventionist may be exposed to X-rays potentially dangerous for his or her health when performing a large number of interventions.

Consequently, given the accuracy needs as well as the necessary X-rays protection, CT-guided robotic systems are gaining more and more attention. CTguided robotic interventions are not new as discussed by Taylor 1. Indeed, Kwoh et al. 22 have done some early work for neurosurgery, Stoianovici et al. [3] on the kidney and they have been followed by other researchers for other organs and procedures. Clinical trials have already been achieved in some cases, but current systems are not well suited for abdominal interventions where the motions and the respiration of the patient create large disturbances.

A general analysis of all existing abdominal procedures is out of the scope of this paper. As an application, we will concentrate on the liver, an organ that is commonly studied because of its importance in numerous pathologies. Furthermore, the internal motions of the liver due to the breathing of the patient makes it a challenging testbed for an image-guided needle insertion robotic system.

\section{Percutaneous Procedures of the Abdomen}

For a clear description of a typical insertion, we decompose the medical gesture in different steps:

1. Localization of the target using imaging devices;

2. Planning of the trajectory of the needle in the images;

3. Selection of an entry point on the abdomen of the patient with an insertion angle;

4. Small incision at this point and beginning of the procedure;

5. While not on target;

a) Insertion of a few centimeters, guided by tactile sensing and synchronized with breathing motion;

b) release of the needle for free motion around the entry point;

c) New image acquisition for checking;

6. End;

7. Target reached.

Currently, completing all these steps takes about half an hour and accuracy up to $10 \mathrm{~mm}$ is considered to be good when the depth is about $150 \mathrm{~mm}$. The first and second steps are often done with pre-operative scans of the patient. As CT-scans provide good tissue differentiation, the planning for needle trajectories 
is straightforward for physicians. The third step is then made by the radiologist who holds the needle inside the scanner and valids the entry point by acquiring images with the CT-scan (see Fig. 11).

During the insertion phase (5a), the radiologist uses the tactile feedback to detect transitions between organs. This important source of information helps him to guide the needle through the different layers of tissue while the image checks allows him to follow a specific angle of attack and precisely reach a structure deep inside the organ.

Mainly, for accuracy issues, protection of the radiologist and the medical staff, and faster interventions, is it clear that a robotized system will improve the previous oper-

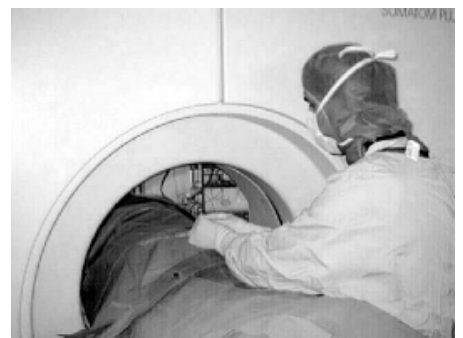

Fig. 1. Check of the needle position during a biopsy under CT guidance ating scheme.

\section{Medical Requirements for a Robotic System}

The observation of a typical biopsy or radio-frequency ablation gives many constraints that are explained in the following section.

\subsection{Patient Safety and Sterilization Issues}

Safety and asepsis are critical requirements. For abdominal access, the motion of the patient and the breathing issue are major difficulties. Real-time compensation of motion by hand is obviously what the radiologist is doing during interventions and such analogy should be employed in the design. Furthermore, the robot must remain motionless with respect to the patient in case of a failure, in order to avoid an undesirable motion or twist of the needle. The sterilization has a direct impact on the selection of the structure and its material characteristics, since nearly all radiological instruments are either placed in safe protective bags or sterilized by auto-clave.

\subsection{Dimensions and Mobility of the Mechanism}

For abdominal interventions, the patient is placed on a translating table that moves in the CT-scan tunnel which is only $700 \mathrm{~mm}$ in diameter. The space dimensions are restricted by the patient build and the table. A patient of stout build fills a large volume of the CT gantry and the available space looks like a $200 \mathrm{~mm}$ radius half-sphere centered on the entry point.

The insertion gesture made by the radiologist is complex and theoretically requires six degrees of freedom to hold and insert the needle. This can be split in two dissociated mobility: first the positioning and orientation of the supporting axis of the needle, second the insertion along the axis of the needle and the self rotation about its axis. The positioning and orientation is done by the robotic 
positioning device described in this paper while the descent and self rotation of the needle will be achieved by a special handling device that is not detailed in this document. Consequently, the required mobility of the positioning device corresponds to three degrees of freedom for positioning the entry point and two additional degrees for orientation of the supporting line of the needle about the entry point.

\section{$2.3 \quad$ CT-Scan Compatibility}

Metal and electrical devices diffuse X-rays. Thus to avoid a distortion of the reconstructed images, these materials must be avoided in the X-rays plane that slices the body of the patient.

\subsection{Exertable Forces and Accuracy}

Several recent papers on bovine liver 4 and pigs liver show that a force of about a few Newtons is applied on the needle during real invivo insertions. Figure 2 shows typical data measured on the insertion axis during in-vivo acquisitions on a liver of a pig through the skin. The force sensor was attached to a needle and inserted by a robot and a radiologist 5]. To achieve a real improvement over manual insertion, an accuracy of at least $5 \mathrm{~mm}$ or better at the tip of the needle is required.

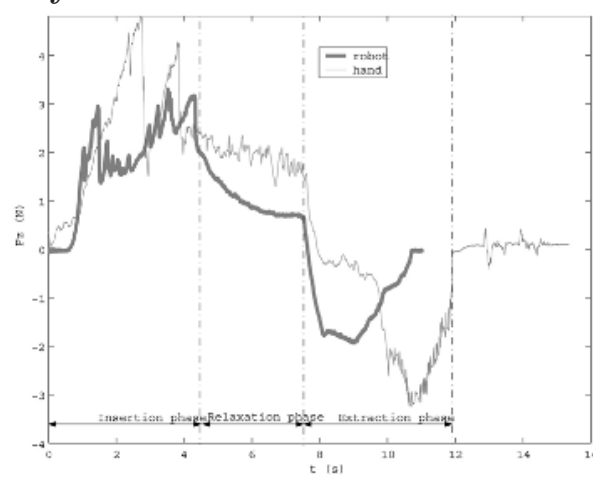

Fig. 2. A typical force measurement on a liver of a pig (by hand and using a robot)

\subsection{Tele-operation and Force Feedback}

We strongly believe that the mradiologist will remain the principal actor of an intervention and a needle insertion robotic system is necessarily tele-operated on a master-slave basis. Currently, the radiologist feels the different tissue layers while driving the needle. This tactile sense must be given through a sensor-based haptic feedback to the master device. A real-time visual representation of the needle inside the body should be beneficial [6] as CT-image slices are not aquired in real-time.

\subsection{Registration of the Needle}

CT-scan allows to compute fiducial-to-image registration thanks to stereotaxic fiducials (see 7]). As the configuration of a robotic system can be fully determined by its forward kinematics, the automatic registration of the needle in the operating space may be an advantage over manual intervention. In fact, even if the needle is not completely visible in the image but the fiducial is, the tip position is known. 


\section{Design and Description of the Robotic System}

\subsection{Answers to the Previous Medical Constraints}

The safety of the patient is undoubtly the most important condition to be favored in the design. Like some recent works done on light robotic systems (see Berkelman et $A l$. or Hong et $A l$. [8/9]) our prototype is also fixed on the abdomen of the patient, so that the motions of the patient and the external motions due to breathing are naturally compensated. Special straps are fastened to the body to avoid involuntary motion of the base support. The robotic device is fixed on the base support. This feature allows to select the best initial configuration according to the intervention objectives. Internal breathing effects can be compensated by synchronisation of the robot and needle driver with the breathing machine.

The size of the robot is small enough to fit in the available space. The weight has been limited to $3 \mathrm{~kg}$ since the robot has to be on the abdomen which is a typical acceptable value. Sterilization of the actuators and sensors through autoclave is very difficult with usual technology, hence a plastic film will be used to protect all electronic devices that can be directly in contact to a human.

The mobility of the platform of the mechanism, supporting the needle-holder, should be a semi-spherical workspace about the entry point. For each accessible point of this workspace, our device may allow two more degrees of freedom for orientation of the platform. The CT-scan compatibility is ensured by choosing a platform that has no metallic parts crossing the X-rays plane.

To decrease the absolute positioning error, a rigid parallel structure is proposed. As a matter of fact, exerted forces are set to $20 \mathrm{~N}$ in our design. Rigidity also provides better force measurements from sensors attached to the platform at the interface with the needle driver. This should help the planned tele-operation force control.

Furthermore, patient-to-robot registration is necessary for visual guidance. For this purpose, stereotaxic fiducials (cubes with metals rods) are used to accuratly estimate the position and orientation of the base of the robot.

\subsection{Structure Description}

The designed structure has three legs, i.e., three serial chains joining the base to the platform. Two opposite legs of the robot are symmetrical chains and form a planar 6-bar linkage. This linkage aims at constraining three degrees of freedom in its plane. Two degrees of freedom remains: the first one is a rotation of the planar 6-bar linkage about the line $\left(\Delta_{1}\right)$ passing through the base (see Fig. 3 ). The second corresponds to the orientation of the platform about the line $\left(\Delta_{2}\right)$. According to the classification of Tsai [10, the system we designed is made of a 6-bar linkage joined to a 4-bar linkage by a common platform, which is a parallel structure. The solution of this kinematic problem is done in closed form using a Local Product Of Exponentials Formalism [11,12. An approximated numerical Jacobian is used to study the workspace and the rigidity of the mechanism since the closed-form solution is not straightforward. 

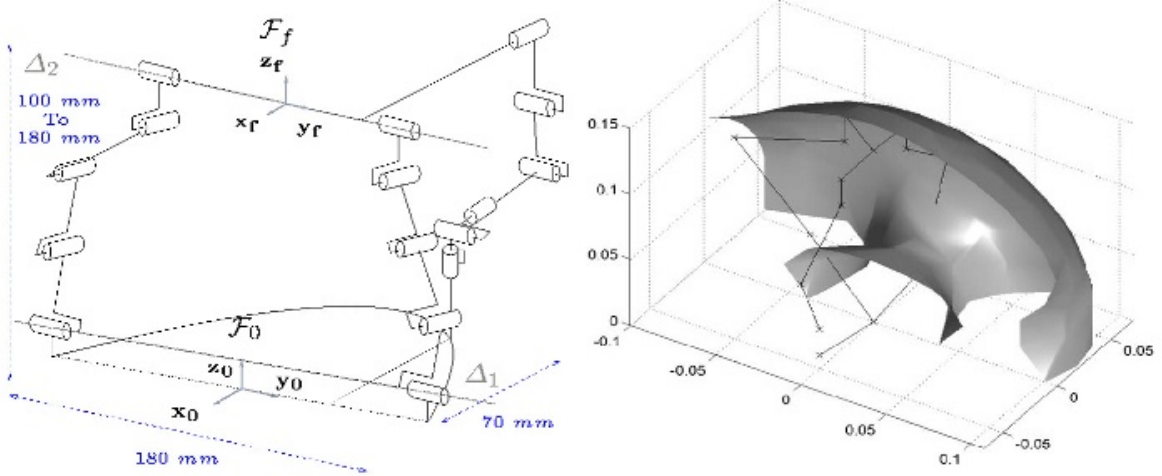

Fig. 3. A kinematic representation of the robot and its reachable workspace

In order to verify the possible motions of the mechanism, a numerical engine in $\mathrm{C}++$ has been build (using Open Dynamic Engine, see Fig. (4) $)$. The simulations show that the kinematics models are well solved. The workspace shown on Fig. 3 has been computed using Matlab and shows only the right half space which is compatible with the previous requirements.

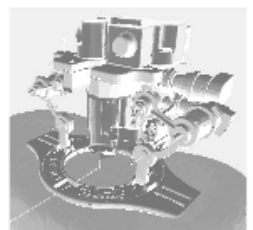

Fig. 4. The simulation environment

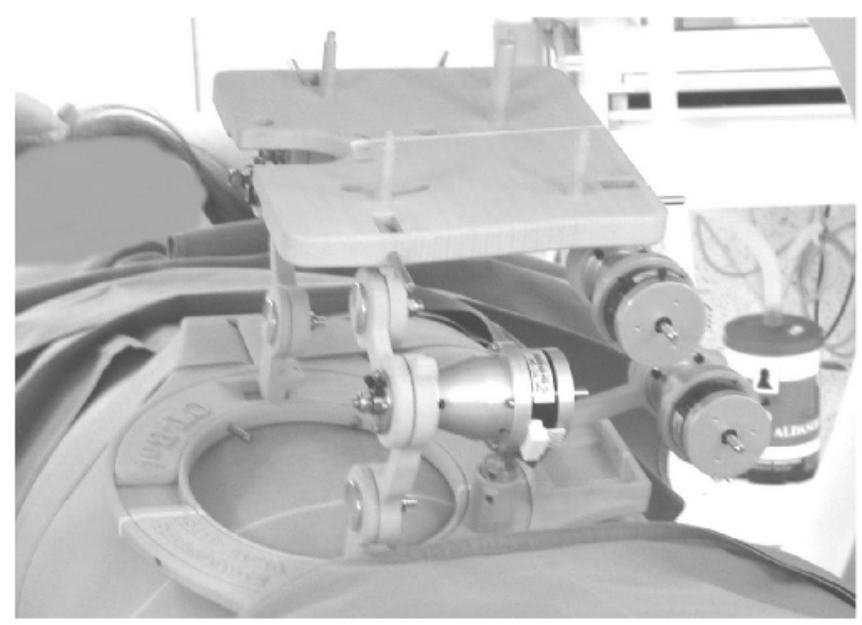

Fig. 5. The robotic system attached to the abdomen, without the needle driver, the cables and the force sensors $(1.5 \mathrm{~kg})$ 


\subsection{Physical Prototype}

Starting from the structural description of the robot (mechanism topology, number of bodies type of joints) the robot is modeled in order to define its kinematics skeleton on a CAD system. The material used to build its links is glass-filled polyamide powder to comply with the CT-Scan imaging requirements (see Fig. [5 for the physical prototype). The mechanical design of the links is done using bearings to limit friction and backlash. A special care is taken to increase structural stiffness of the system. This system is designed to maintain a force of $20 N$ applied to the platform. Each actuation unit comprises a gear housing specifically designed for this application, an Harmonic Drive reduction gearing, an ultrasonic motor and an incremental encoder.

Three force sensors (Sensotec load cells) are symmetrically positioned on the needle driver unit that is attached under the platform. These devices give the force applied along the insertion axis. An haptic interface (Sensable 1.5 PHANToM) is currently used for the feedback needs. The prototype is registered in the CT-scan using stereotaxic fiducials made of $30 \times 30 \times 40 \mathrm{~mm}$ plastic cubes with metal rods (see [13]). The fiducial cubes made for the robot registration are attached to its base (see Fig. 6). For now, an accuracy recontruction of un-

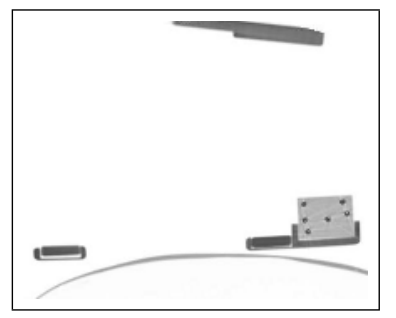

Fig. 6. CT-scan of a fiducial cube attached to the robot der $1 \mathrm{~mm}$ in position is obtained using $512 \times 512$ image with $0.5 \mathrm{~mm}$ scaling factors.

\subsection{Current Work and Limits}

The accuracy of the guidance depends on the fastening of the supporting base. This point is under work and a first solution currently explored is based on a deflating bag. It should hold tight the support to the patient while allowing the breathing motion. The current control scheme is position-based and further improvements have to be done for automatic registration of the system. A navigation-like feature (point and click paradigm) is currently implemented in order to help the radiologist to do the initial placement of the needle.

\section{Conclusion}

This paper presents the strong requirements on a safe robotic assistant for percutaneous procedures on the abdomen under CT-guidance. A novel five degrees of freedom parallel structure is described, intended to help practitioners for guiding and orienting a needle driver. A prototype has been built and is currently tested in a position-based control scheme. Registration of the robot using stereotactic fiducials will allow automatic guidance. 


\section{References}

1. Taylor, R.H.: Medical robotics and computer aided surgery. In: Proceedings of Medical Image Computing and Computer-Assisted Intervention (MICCAI). (2002) Web PDF Hands-out of presentation.

2. Kwoh, Y.S., Hou, J., Jonckheere, E., Hayati, S.: A robot with improved absolute positioning accuracy for CT guided stereotatic brain surgery. IEEE Transactions on Biomedical Engineering 35 (1988) 153-160

3. Stoianovici, D., Whitcomb, L.L., Anderson, J.H., Taylor, R.H., Kavoussi, L.R.: A modular surgical robotic system for image guided percutaneous procedures. In: Proceedings of Medical Image Computing and Computer-Assisted Intervention (MICCAI), Cambridge, MA (1998) 404-410

4. Simone, C., Okamura, A.M.: Modeling of needle insertion forces for robot-assisted percutaneous therapy. In: Proceedings of the IEEE International Conference on Robotics and Automation (ICRA), Washington, DC, USA (2002) 2085-2091

5. Maurin, B., Barbe, L., Bayle, B., Zanne, P., Gangloff, J., de Mathelin, M., Gangi, A., Forgionne, A.: In vivo study of forces during needle insertions. In: Proceedings of the Medical Robotics, Navigation and Visualisation Scientific Workshop, Remagen, Germany (2004)

6. Gerovichev, O., Marayong, P., Okamura, A.: The effect of visual and haptic feedback on manual and teleoperated needle insertion. In: Proceedings of Medical Image Computing and Computer-Assisted Intervention (MICCAI), Tokyo, Japan (2002) 147-154

7. Susil, R.C., Anderson, J.H., Taylor, R.H.: A single image registration method for ct guided interventions. In: Proceedings of Medical Image Computing and Computer-Assisted Intervention (MICCAI), Cambridge, GB (1999) 798-808

8. Berkelman, P.J., Cinquin, P., Troccaz, J., Ayoubi, J.M., Létoublon, C., Bouchard, F.: A compact, compliant laparoscopic endoscope manipulator. In: Proceedings of the IEEE International Conference on Robotics and Automation (ICRA), Washington DC, USA (2002) 1870-1875

9. Hong, J., Dohi, T., Hashizume, M., Konishi, K., Hata, N.: An ultrasound-driven needle-insertion robot for percutaneous cholecystostomy. Physics in Medicine and Biology 49 (2004) 441-455 IOP Publishing Ltd.

10. Tsai, L.W.: Mechanism Design : enumeration of kinematic structures according to function. Mechanical Engineering series. CRC Press (2001)

11. Murray, R.M., Li, Z., Sastry, S.S.: A Mathematical Introduction to Robotic Manipulation. CRC Press (1994)

12. Yang, G., Chen, I.M., Lim, W.K., Yeo, S.H.: Design and kinematic analysis of modular reconfigurable parallel robots. In: Proceedings of the IEEE International Conference on Robotics and Automation (ICRA), Detroit, MI (1999) 2501-2506

13. Maurin, B., Doignon, C., de Mathelin, M., Gangi, A.: Pose reconstruction from an uncalibrated computerized tomographic device. In: Proceedings of the IEEE International Conference on Computer Vision and Pattern Recognition (CVPR). Volume 1. (2003) 455-460 